\title{
CAPnet: A Defense Against Cache Accounting Attacks on Content Distribution Networks
}

\author{
Ghada Almashaqbeh $^{1}$, Kevin Kelley ${ }^{2}$, Allison Bishop ${ }^{1,3}$, and Justin Cappos ${ }^{4}$ \\ 1 Columbia University, NY, USA \{ghada, allison\}@cs.columbia.edu \\ 2 CacheCash Development Company, CA, USA kelleyk@kelleyk.net \\ 3 Proof Trading, NY, USA \\ 4 New York University, NY, USA jcappos@nyu.edu
}

\begin{abstract}
Peer-assisted content distribution networks (CDNs) have emerged to improve performance and reduce deployment costs of traditional, infrastructure-based content delivery networks. This is done by employing peer-to-peer data transfers to supplement the resources of the network infrastructure. However, these hybrid systems are vulnerable to accounting attacks in which the peers, or caches, collude with clients in order to report that content was transferred when it was not. This is a particular issue in systems that incentivize cache participation, because malicious caches may collect rewards from the content publishers operating the CDN without doing any useful work.

In this paper, we introduce CAPnet, the first technique that lets untrusted caches join a peer-assisted CDN while providing a bound on the effectiveness of accounting attacks. At its heart is a lightweight cache accountability puzzle that clients must solve before caches are given credit. This puzzle requires colocating the data a client has requested, so its solution confirms that the content (or at least an amount of data within a pre-configured bound) has actually been retrieved. We analyze the security and overhead of our scheme in realistic scenarios. The results show that a modest client machine using a single core can solve puzzles at a rate sufficient to simultaneously watch dozens of $1080 \mathrm{p}$ videos. The technique is designed to be even more scalable on the server side. In our experiments, one core of a single low-end machine is able to generate puzzles for 4.26 Tbps of bandwidth - enabling 870,000 clients to concurrently view the same $1080 \mathrm{p}$ video. This demonstrates that our scheme can ensure cache accountability without degrading system productivity.
\end{abstract}

\section{Introduction}

Online content distribution has grown dramatically over the last decade. Video streaming, in particular accounts for more than $60 \%$ of today's Internet traffic and it is expected to exceed $80 \%$ by 2022 [3]. To meet this huge demand, content publishers typically employ solutions that distribute the load among geographically dispersed caches. Among these solutions, infrastructure-based content delivery networks (CDNs) have proven effective $[1,4]$. But as load continues to 
grow, pressure to improve performance and reduce cost has led to experimentation with more sophisticated topologies [13,18].

Peer-assisted CDNs have evolved to reduce these costs and also to allow access to lower latency peers. In this model, centralized services are supplemented with the resources of end users, or peers [12,24]. By allowing anyone to join, and exchanging service for a payment, this paradigm creates robust, performant, and flexible systems. In addition, peer-assisted solutions can extend the network coverage of infrastructure-based CDNs, scale more easily with demand, and when managed carefully, offer a good quality of service for end users [24]. Several commercial CDN providers have built products that employ this approach, such as Swarmify [8], Velocix [9], and Akamai NetSession [2].

However, peer-assisted work models are vulnerable to cache accounting attacks $[11,19]$, in which a cache and client collude to defraud the content publisher by claiming to have transferred data (and claiming payment) when no actual work has been done. This is a particular problem in content distribution applications that do not require subscription fees from clients, such as ad-funded video streaming [10], or services that allow a client to play content on multiple devices under one subscription [6]. Some defenses against these attacks have been proposed [11,22], but they do not work in typical peer-to-peer scenarios, where untrusted, anonymous nodes serve as caches.

In this paper, we introduce CAPnet, the first technique that lets untrusted caches, such as peers with unknown computational and latency characteristics, join a peer-assisted CDN while providing a bound on the effectiveness of accounting attacks. Our key innovation is a lightweight cache accountability puzzle that clients must solve before caches are given credit. The puzzle solution serves as a content retrieval confirmation assuring publishers that the claimed content transfer (or at least an amount of data within a pre-configured bound) has taken place.

For each service request in CAPnet, the publisher generates a puzzle that a client must solve by processing the data chunks retrieved from caches (each of which is encrypted with a request-specific key). Solving this puzzle requires the client to sequentially touch small pieces of these chunks in an unpredictable order. Because of this unpredictability, the communication overhead of generating the solution without having the data colocated is significant. Combined with the use of a completion mask, a secret that is used to conceal an encrypted data chunk until it has been completely transferred, this processing pattern forces colluding parties to expend bandwidth similar to retrieving the content, and thus removing any motivation to cheat.

Equally important for its use in practical applications, CAPnet does not sacrifice efficiency for enhanced security. Its tools are built on computationallylight operations (symmetric encryption and hashing). CAPnet is also designed to be scalable; while a client needs to process a large portion of the retrieved content when solving a puzzle, a publisher needs only to process a small, serverconfigurable number of pieces to generate a challenge. Because of this asymmetry, 
our scheme can meet the deployment demands of large-scale content distribution applications.

To demonstrate that CAPnet is effective at mitigating cache accounting attacks, we configure the system parameters based on an analysis of the bandwidth cost incurred by malicious puzzle-solving strategies. Our analysis shows that the publisher can ensure that a malicious actor must expend a substantial amount of bandwidth, even given unrealistically strong assumptions about the malicious actors capabilities. To evaluate CAPnet's efficiency, we experimentally evaluate the computational overhead of our scheme under various configurations. The benchmark results show that a modest client machine can solve puzzles at a rate sufficient to confirm the retrieval of around $170 \mathrm{Mbps}$, which is enough to watch more than 30 1080p quality videos simultaneously. Even a single core low-end publisher machine can generate enough puzzles to support 870,000 simultaneous views of the same video.

\section{Related Work}

To orient readers to current state-of-the-art defenses for cache accounting attacks, this section reviews prior work done in this area. We also present information about a related topic — proofs of data storage - and discuss why this proof paradigm is not applicable to cache accounting attacks.

Cache accountability in peer-assisted CDNs. One technique used in peerassisted CDNs is to rely on the peers themselves to report statistics about content delivery. For example, clients in Akamai Netsession [2] share reports about their upload and download activity, and this information is used to manage network resources. Even some systems that exchange service for monetary rewards, e.g., [21], rely on these types of reports to track the service contributions of peers in order to pay them accordingly. However, in such an open environment that allows anyone to join, peers may fabricate these accounting reports. This has been confirmed through empirical studies [11,19].

Consequently, specialized cache accountability defenses work to address this issue by making clients commit to these activity logs. This is done by requiring participants to maintain tamper-evident logs, and cryptographically sign all messages sent to the network. The participants periodically exchange these logs with a verifier. The verifier in turn checks the consistency of the reported information and performs anomaly detection to identify cheating based on a protocol reference implementation. The repeat and compare scheme [20] utilizes this technique to address the problem of corrupted content distribution. PeerReview [17] employs a similar approach to detect Byzantine faults. And RCA (Reliable Client Accounting) [11] exploits such logs to address the same collusion problem we describe in this paper. However, this approach cannot prevent colluding parties from fabricating consistent and valid-looking log reporting content transfers that did not take place. Thus, cheating clients and caches can still collude to collect rewards for work they did not perform. 
A prior bandwidth puzzle-based defense, proposed by Reiter et al. [22], works by issuing challenge puzzles to all caches and clients (which must have known computational abilities and communication latency) that possess the content. These parties have to solve the issued puzzles over the retrieved content in a short period of time to receive payment. This scheme has several downsides when compared to our approach. First, every time a new party retrieves the content, puzzles must be solved by all peers that have a copy of this content, even those uninvolved in the transfer. Second, the security of the scheme is based on knowing a bound for the attacker's hashing power, which is used to quantify the number of challenge puzzles that must be presented within a time window. Third, the latency of peers must also be known for the scheme to resist cheating. This latency constraint may cause peers to lose their rewards in the event of lost or delayed messages. In addition, an attacker that can fool others into believing they have high latency can cheat because she has more time to solve puzzles (including those that were supposed to be solved by other caches).

Relation with data possession proofs. Several works in the literature have tackled a related problem - how to prove that a server to which a client has outsourced files is actually storing those files, e.g., ensuring correct data storage in the cloud [23]. Solutions to this problem include proofs-of-retrievability [14], proofs of data possession [16], and proofs-of-storage [15]. Such proof systems, at first glance, could be viewed as potential defenses against cache accounting attacks. That is, a publisher can ask a client to prove the storage of a local copy of the retrieved content. However, this does not confirm that caches have served the content. These colluding caches can generate valid proofs of storage for any client because they store the full raw content. Similarly, a client that retrieves some content only once can produce valid proofs, for itself or others, for all future requests that ask for the same content. While useful, these proof systems are not applicable for fighting cache accounting attacks.

\section{CAPnet Design}

CAPnet defends against cache accounting attacks by both mandating proof of delivery, and making honest choices more profitable than cheating. In this section, we describe how the design of CAPnet manages this defense. We start by defining the work environment for content distribution systems our scheme targets, then provide a high level view of the primary operations, after which we present the technical details of these operations.

\subsection{Work Environment Model}

In the incentivized, peer-assisted content distribution systems that CAPnet targets, there are three participant types: publishers, caches, and clients. A publisher owns content, e.g., videos or software packages, that clients want to retrieve. A publisher hires caches to distribute this content in exchange for rewards, such 
as monetary incentives, which are tied to the amount of service these caches provide. Each cache is defined by its IP address, which the publisher monitors to detect Sybils. When a cache joins a publisher's network, it gains access to the content to be served, which we assume to be divided into equally-sized data chunks. A client request can fetch a range of $n$ chunks within the object, e.g., movie, it wants to retrieve.

During the content distribution process, a publisher acts as a dispatcher assigning caches to fulfill client requests. Therefore, clients must contact the publisher first, asking for $n$ data chunks, to obtain the list of $n$ caches that will provide the service. The publisher selects this set randomly such that each cache will serve a single data chunk among the requested set.

As will be shown shortly, confirming that the content has been retrieved is done over individual content requests. In other words, even if the client wants to retrieve a large object, e.g., a movie of size $1 \mathrm{~GB}$, it computes the retrieval confirmation over each $n$ chunks separately. Such an approach reduces the amount of memory that CAPnet requires, e.g., for $n=4$ and a chunk size of $1 \mathrm{MB}$, a client/publisher would need only a $4 \mathrm{MB}$ storage to hold the chunks needed for processing any request.

CAPnet enables the publisher to set a bound on the amount of bandwidth the attacker must expend, with respect to the original content amount, which we call the $\delta$-bound. So, for $4 \mathrm{MB}$ of content, a 0.75 -bound attacker in our scheme is expected to expend $3 \mathrm{MB}$ to provide a valid content retrieval confirmation for a content request. The $\delta$-bound is controlled by the number of rounds in the cache accountability puzzle of CAPnet. The larger $\delta$, the larger the computation cost of generating and solving this puzzle. Therefore, system designers need to configure this parameter based on the desired security-efficiency trade-off they want to achieve.

Lastly, we work in the random oracle model, where hash functions are modeled as random oracles. We also work in the ideal cipher model, where a block cipher is modeled as a random permutation. In addition, we deal with efficient adversaries that cannot break secure cryptographic primitives, such as AES, SHA256, and pseudorandom functions (PRFs), with non-negligible probability.

\subsection{Overview}

CAPnet consists of a set of actions integrated into the content delivery process. Collectively, these actions demonstrate that the required bandwidth amount was actually expended, even in the face of malicious, colluding client and caches. In what follows, we provide an intuitive discussion of these actions to highlight the motivation behind the design (a rigorous security analysis of how these actions defend against cache accounting attacks is found in the next section).

As shown in Figure 1, to request content in the CAPnet model, a client retrieves a request bundle from the publisher that enables the retrieval of $n$ data chunks (steps 1, 2, and $\mathbf{3})^{5}$. This bundle stipulates which caches to contact, and

\footnotetext{
${ }^{5}$ If the content has more than $n$ chunks a client will send several requests.
} 


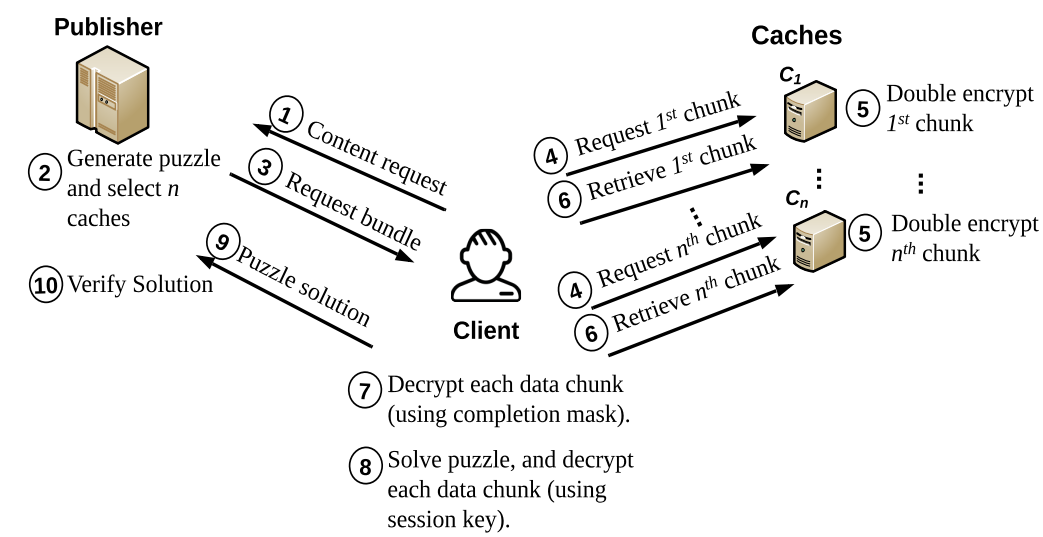

Fig. 1: CAPnet integration in content distribution ( $n$ is the number of caches selected for a service request).

includes the client's IP and a request number. In addition, the bundle contains a puzzle, that when solved, enables the client to prove that the requested chunks (or at least an amount of data within the $\delta$-bound) were indeed retrieved.

The client contacts caches, possibly in parallel, and provides the request bundle that instructs each cache what specific data chunk to serve (step 4). Each cache will encrypt its data chunk with a unique per-request key, and additionally encrypts the produced ciphertext using a fresh per-request completion mask (step 5). The double encrypted chunk, appended with the completion mask, is then delivered to the client (step 6). Once all chunks are received, the client decrypts each chunk using the completion mask as the decryption key (step 7), and solves the puzzle using the single-layer encrypted chunks (step 8). With the puzzle solution the client can decrypt the data chunks to obtain the raw content (step 8), and confirm to the publisher that these chunks were retrieved (steps 9 and 10).

When a cache begins serving content for a publisher, they establish a shared secret called a master key. Along with the request number, both parties use this key to non-interactively generate a fresh per-request session key that is used to encrypt the data chunk the cache will serve. Since this key is unique and cannot be distinguished from random ${ }^{6}$, each request returns a different, random looking, encrypted $\operatorname{chunk}^{7}$, even if the raw content is the same. Also, since the session key is a secret known only to the publisher and that cache, a malicious cache does not know the encrypted content that an honest cache would serve.

In addition, CAPnet ensures that a client retrieves the entire encrypted chunk before it can start solving the puzzle. To do this, for each request a cache selects a random completion mask, i.e., a random key, that is used to encrypt the chunk ciphertext. In other words, the cache adds a second layer of encryption using the

\footnotetext{
${ }^{6}$ This is by the security of PRFs.

${ }^{7}$ Recall that we work in the ideal cipher model.
} 
completion mask as the encryption key. The cache appends the completion mask to the transmitted, double encrypted chunk. Thus, the client has to download the entire chunk before being able to unmask any part of it.

A puzzle is computed by processing small (e.g., 16 byte long) pieces of the (single-layer) encrypted chunks. Starting at a randomly selected piece in the first chunk, one computes the hash of this piece and maps it to a piece index in the second data chunk. In the random oracle model, this mapping randomly jumps to a piece in the second chunk. The hash is now computed over the previous hash and the second piece and is used to select another piece in the third chunk, and so on. Once the data chunk from each cache is visited, this completes a round. The next round is begun by mapping the last hash of the prior round to a piece index in the first chunk. This continues for the number of rounds chosen by the publisher to achieve the desired $\delta$-bound.

The publisher and the client compute the puzzle in slightly different ways. The publisher randomly chooses a "starting piece" in the first chunk and computes one puzzle to produce a challenge for the client. This challenge does not contain any information about the starting piece. Hence, the client will attempt to solve the challenge by computing candidate, or trial, puzzles initiated at various starting pieces in the first chunk until the solution is found. This forces the client to process a $\delta$ portion of the content before finding the solution.

Increasing $\delta$ strengthens the security guarantees of CAPnet by causing malicious parties to retrieve more content, but also increases the computation cost for publishers and honest clients as larger number of rounds are needed. Caches, on the other hand, have uniform computational cost independent of the $\delta$-bound.

\subsection{Design Details}

We now describe the CAPnet actions in more detail, including puzzle generation, solving, and verification.

Puzzle Generation The publisher generates a challenge puzzle based on the data chunks a client wants to retrieve. Figure 2 depicts this action through a clarifying example involving two data chunks. In this figure, $L$ stands for location, $H$ stands for hashing, $E$ stands for encryption, $\|$ is a concatenation operation, and the arrows indicate the sequence of pieces selected when computing a puzzle.

As shown, a puzzle starts at the first data chunk and proceeds by processing a number of small data pieces selected at random. Given that a puzzle round processes encrypted pieces, the publisher encrypts the piece selected at each step. It then hashes the encrypted piece along with prior hash or location value. The output hash is mapped to a piece index within the next chunk (or the first chunk is this is the beginning of a new round).

This computation pattern imposes three aspects. First, each location value encapsulates all encrypted pieces processed so far, which enforces sequential computation of the puzzle. Second, processing encrypted pieces prevents any correlation between puzzles generated for different requests, even if they are for 


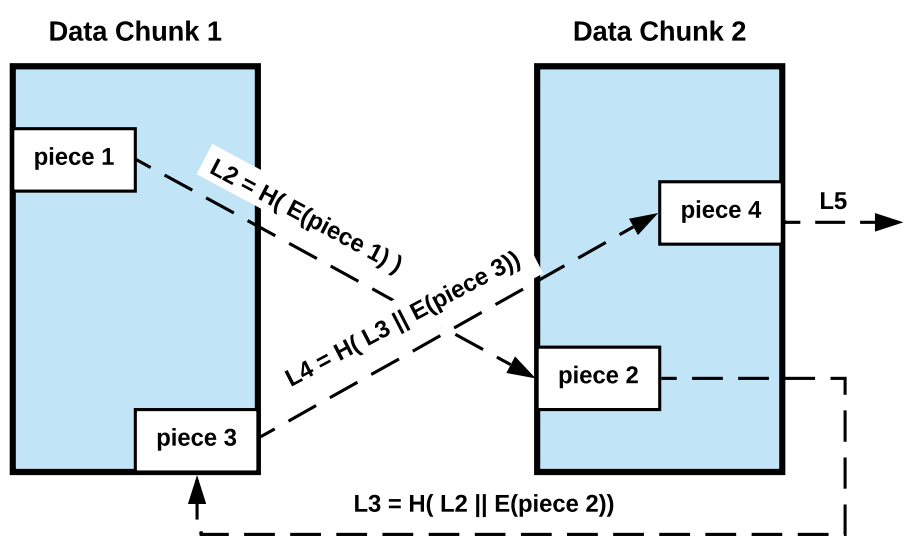

Fig. 2: An example of puzzle challenge generation with two chunks and two rounds. The puzzle challenge is $H\left(L_{5}\right)$ and its solution is $L_{5}$.

the same raw content. Third, touching all data chunks in a round robin order makes all chunks contribute equally in solving a puzzle. This ensures that a puzzle solution is computed over all chunks, confirming their retrieval.

Once the puzzle computation is completed, which happens when the designated number of rounds is reached, the publisher uses the hash of the last location, i.e., $H\left(L_{5}\right)$ in the figure, as the challenge, and it asks the client to return the preimage of this hash, i.e., $L_{5}$. This is done without revealing piece $e_{1}$. Instead, the client tries all data pieces in the first chunk. Hiding the starting piece causes the client to touch a large percentage of the pieces in each chunk while enabling the publisher to touch very few. This minimizes the computational load for publishers, allowing them to process a large number of client requests concurrently.

The technical details of this process are captured by Algorithm 1. In this algorithm, $D_{j}$ is the $j^{\text {th }}$ data chunk, piece $e_{i}$ is the $i^{\text {th }}$ data piece in a data chunk, $R_{\text {puzzle }}$ is the number of puzzle rounds, and piecestotal is the total number of pieces in any data chunk. The data pieces inside a chunk are referenced using their indices, where the first piece has an index 0 , the second has an index 1 , and so on. We use index $(\cdot)$ to denote the index of a given piece.

Algorithm 1 shows two phases: an initialization phase, and a challenge preparation phase. The initialization phase is needed to allow caches and the publisher to agree on the encryption setup. We use AES in the counter mode (AES-CTR) for encryption because it allows a publisher to encrypt any individual data piece without encrypting the whole data chunk. To generate identical encrypted data, the publisher and each cache $C_{j}$ must generate the same session key $k_{j}$ and the initial value of the AES-CTR counter $c t r_{j, \text { initial }}$.

Generating the AES-CTR counter and session key is done using a one time setup without any per-request interaction between the publisher and cache. As mentioned before, a cache $C_{j}$ shares a master key with the publisher that they 


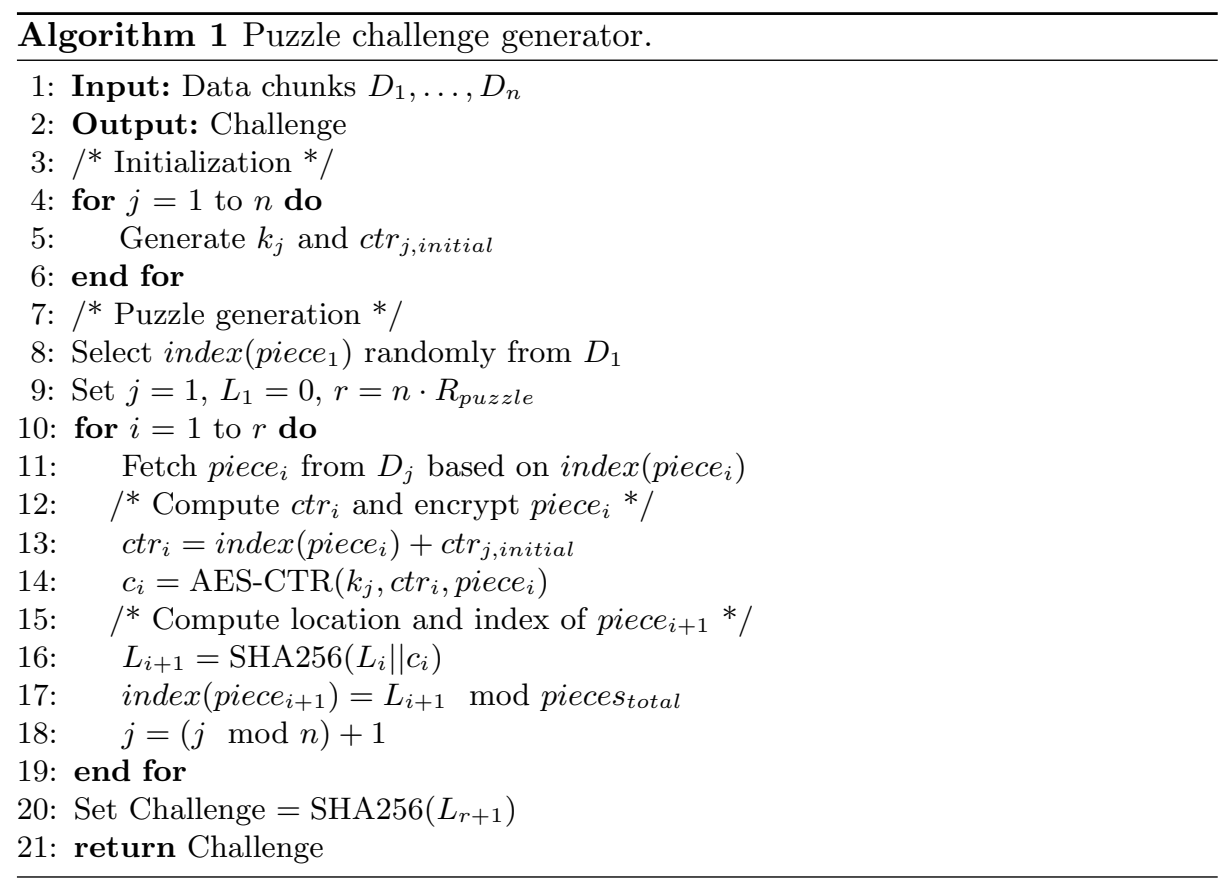

both use to derive any future session key $k_{j}$. This is done by means of a pseudorandom function (PRF) keyed with this master key, and evaluated over the request number and the client IP to output $k_{j}$. The same PRF idea, but keyed with the session key, is used to generate $c t r_{j, \text { initial }}$. Accordingly, in the initialization phase, the publisher generates all keys and counters for all data chunks that will be used by the caches involved in the service session.

The puzzle generation phase proceeds as described previously. After selecting a piece index at random from the first data chunk, the publisher proceeds by computing the location of the next piece (line 16), and mapping this location to a piece index (line 17). The new location computation requires encrypting the prior piece, which in turn requires computing the correct AES-CTR counter value (line 13). By doing so, the publisher produces the same ciphertext of the selected piece that a cache will produce. The aforementioned process is repeated for the required number of iterations, as found in lines 10-19. Lastly, the algorithm outputs the hash of the last location as the puzzle challenge.

After the puzzle is generated, the publisher informs the client about the puzzle challenge it has to solve as part of the request bundle mentioned earlier. To allow the client to decrypt the retrieved data chunks, the publisher can either provide the keys in response to the puzzle solution reported by the client, or simply encrypt all session keys using the puzzle solution and share the ciphertext as part of the request bundle. Either way, once the client solves the challenge puzzle it can recover these keys and decrypt the received data. 
Puzzle Solving The client receives the puzzle challenge, along with the cache contact information, within the request bundle sent by the publisher. With this bundle, the client can start the content retrieval process, where it connects with the listed caches and requests the specified data chunks. Caches will deliver double-layer encrypted chunks, with the completion mask appended to each chunk. The client uses the completion mask as the decryption key to remove the second encryption layer of the chunk. By repeating this process for all chunks, the client obtains the single-layer encrypted data chunks.

The client can now perform the second action in the CAPnet process solving the challenge puzzle. It uses a similar algorithm to the one used by the publisher with three differences. First, since the client retrieves encrypted data chunks from the caches, it does not encrypt the data pieces before applying the hash, thus skipping lines 4-6 and 13-14 in Algorithm 1. Second, since the client does not know the starting piece, it computes a puzzle for every piece in the first data chunk until the correct solution is found. In other words, it repeats lines 9-11 for each candidate starting piece. And third, once the client solves the puzzle, the output is the puzzle solution, which is the last location in the correct puzzle.

Puzzle Verification The last action in the CAPnet process is verifying the correctness of the reported puzzle solution. While it would be possible to keep a record of the puzzle challenges and their solutions for each client, we devise a computationally-lightweight technique that does not require maintaining any per-client state.

In this technique, the publisher generates a unique secret token for each content request. This is done by evaluating a secret PRF over the concatenation of the request number and the client IP. The publisher then encrypts the secret token using the puzzle solution, and sends the encrypted token to the client as part of the request bundle. Once the client solves the puzzle, it can decrypt the token and send it back to the publisher along with the request number. The publisher can simply evaluate the secret PRF over the request number and the client IP, and thus, verifies whether the output equals to the token value reported by the client. This enables the publisher to quickly check the correctness of a puzzle solution.

\section{Security Analysis}

In this section, we analyze the effectiveness of CAPnet in fighting cache accounting attacks. We begin by outlining the setup of this analysis, after which we discuss how the security of CAPnet changes as the design parameters change.

\subsection{Setup}

The analysis setup defines how we model our adversaries, and explains the security properties that CAPnet is designed to achieve. The set of notations that this analysis uses is shown in Table 1. 
Table 1: Notations.

\begin{tabular}{|c|c|}
\hline Symbol & Meaning \\
\hline$n$ & Total number of caches in a service session. \\
\hline $\mathbf{C}_{m}$ & Set of malicious caches in a service session. \\
\hline$m$ & Number of malicious caches in a service session, where $m \leq n$. \\
\hline$h_{\text {size }}$ & Hash output size. \\
\hline chunk $_{\text {size }}$ & Data chunk size. \\
\hline piece $_{\text {size }}$ & Data piece size, where piece $_{\text {size }} \leq \frac{h_{\text {size }}}{m}$. \\
\hline pieces $s_{\text {total }}$ & Total number of pieces in a data chunk, where pieces $_{\text {total }}=\frac{\text { chunk }_{\text {size }}}{\text { piece }_{\text {size }}}$. \\
\hline$R_{\text {puzzle }}$ & Number of puzzle rounds. \\
\hline$Y$ & $\begin{array}{l}\text { A random variable that represents the number of pieces a malicious } \\
\text { puzzle solver retrieves. }\end{array}$ \\
\hline $\mathbb{E}[Y]$ & The expectation of $Y$. \\
\hline$\delta$ & $\begin{array}{l}\text { The ratio between the bandwidth amount a malicious puzzle solver } \\
\text { would spend and the amount that an honest solver would use. This is } \\
\text { computed as } \delta=\frac{\mathbb{E}[Y]}{n \cdot \text { pieces }_{\text {total }}} \text {. }\end{array}$ \\
\hline
\end{tabular}

Adversary Model. We consider a client colluding with a set of $m \geq 1$ caches $^{8}$. (If a client does not collude with any cache, it must retrieve all the data chunks to solve the puzzle just like an honest client.) This collusion can be modeled as an interaction between two parties: the client and a collective entity $\mathbf{C}_{m}$. Any cache $C_{j}$ in $\mathbf{C}_{m}$ can pool all encrypted data chunks from the rest of the malicious caches at a very low cost. That is, given that each cache has a full copy of the raw content, $C_{j}$ needs only the session keys of these caches to produce their encrypted data chunks locally. When we say that a client retrieves data pieces from $\mathbf{C}_{m}$, we mean that this client is interacting with the cache that pooled the data chunks.

In order to have a strong bound on attacker capabilities, we consider an attacker with full knowledge about the piece distribution across all the trial puzzles a client will compute. In other words, the attacker knows the selection frequency of data pieces, i.e., how many times a piece has been processed by all puzzles, in all chunks rather than just in the puzzles for which this attacker has enough prior pieces. The attacker may use this information to retrieve the most frequent pieces when solving the challenge puzzle.

Despite the hash function being modeled as a random oracle with a uniform and random output, this piece frequency still matters. Suppose that we have a

\footnotetext{
${ }^{8}$ All these caches are different, i.e., not Sybils run on the same machine. This is due to the assumption that a publisher monitors the IP addresses of its caches to detect Sybils.
} 
chunk composed of 4 pieces that are randomly chosen to be in 4 trial puzzles. Over $90 \%$ of the time one of these pieces is chosen at least twice (only about $9 \%$ of random draws of 4 items choose one from each). On average more than 1 piece is likely not to be chosen for any trial puzzle. An intelligent attacker would choose the piece used in the most trial puzzles since it gives the greatest chance to solve the challenge. So, accounting for the fact that the actual frequencies are not uniform, even with a random function, more accurately models the attacker's capabilities. Furthermore, providing the attacker perfect information about these actual frequencies implies that the security bound we infer will be conservative.

The client and $\mathbf{C}_{m}$ want to solve the puzzle while expending as little bandwidth as possible. In quantifying this cost, we compute the download bandwidth consumption for the colluding group.

Our adversary model is subject to the following assumptions:

A1. Secure cryptographic primitives. Adversaries cannot efficiently break the basic cryptographic building blocks (SHA256, AES, and PRFs) with non-negligible probability.

A2. Clients do not already possess the content. At the beginning of a service session, a client does not have a copy of the content it will request. This can be achieved by having publishers track which clients have retrieved which content. However, even if this assumption is violated, the client still must retrieve data chunks from honest caches in order to solve a puzzle, leading to the retrieval of at least $\delta=\frac{n-m}{n}$ of the requested chunks.

A3. Free adversarial metadata communication. It is difficult to know the minimal size of information adversaries would need to communicate when coordinating the puzzle solving process. Therefore, we will just assume that such costs are free from a bandwidth standpoint and only count data piece transmission. While it ignores some cost, this makes the overhead numbers conservative in that real attackers will incur more cost than what we predict.

A4. Content is already compressed. The raw content distributed by caches is already compressed. As such, a malicious cache who intends to compress the raw content before encrypting it will only save a very small bandwidth amount.

In addition to the above, and as mentioned previously, we work in the random oracle model (i.e., hash functions are modeled as random oracles), and in the ideal cipher model (i.e., block ciphers are modeled as random permutations).

An intelligent client and $\mathbf{C}_{m}$ collaborate to solve the puzzle while transferring the least amount of data possible. In this collaboration, the client receives encrypted chunks only from honest caches, while $\mathbf{C}_{m}$ produce all encrypted chunks of malicious caches by pooling their session keys as explained previously. (We consider that $\mathbf{C}_{m}$ have pooled the data to simplify our analysis.) The strategy then will have a solver, either the client or $\mathbf{C}_{m}$, that attempts to solve the puzzle using the chunks it has in addition to information it requests from the second party, whichever of the client or $\mathbf{C}_{m}$ is not the solver. The second party acts as a piece provider which sends pieces or hashes (i.e., piece locations computed in 
a puzzle round) to the solver. For reasons we will see shortly, pieces make more sense for the attacker to transmit.

As our analysis will show, the client and $\mathbf{C}_{m}$ can decide in advance which party will play which role based on which option incurs the least bandwidth cost. This decision depends on the number of malicious caches $m$. If this number is less than half, i.e., $m<\frac{n}{2}$, it is more efficient for the client to act as the solver. If it is greater than half, it is more efficient for the client to let $\mathbf{C}_{m}$ solve the challenge puzzle. If the number of caches is exactly half, it is equally efficient regardless of who is the solver.

Other attack strategies may involve attacking the cryptographic primitives used in CAPnet design. This may include trying to find the preimage of the puzzle challenge by inverting the hash, or predicting the session keys used by honest caches to produce their encrypted chunks locally by a colluding cache. By the security of the underlying cryptographic primitives CAPnet employs, i.e., the use of secure PRFs and first-preimage resistant hash functions, such strategies will succeed with negligible probability.

It should be noted that the above list is not known to be comprehensive. There could be other attack strategies outside the analysis presented in this section (although we are not aware of any of such strategies). Performing a rigorous analysis over the full potential threat space is an open problem for future work.

Security Goal. The goal is to ensure that a malicious puzzle solver cannot solve the challenge puzzle in CAPnet unless it expends, on average, a bandwidth amount equivalent to retrieving at least $\delta$ portion of the content. This means that the colluding group is expected to expend a total of $n \delta c h u n k_{\text {size }}$ bandwidth units. So for $\delta=0.95$, the attacker has an expected value of $95 \%$ of the bandwidth cost even if all metadata overhead are ignored. System designers may set the value of $\delta$ based on the security-efficiency trade-off they want to achieve. A larger $\delta$ value provides stronger security guarantees, but also increases the computational cost of generating and solving puzzles.

It is not practical to have $\delta=1$ unless the publisher touches every piece of the requested chunks. Since each chunk must be encrypted with a fresh key, this cost is prohibitive. In fact, if the publisher is willing to touch (and encrypt) every piece, it is simpler to compute the hash of the encrypted chunks, and use this hash as the content retrieval confirmation that a client has to compute. However, this would greatly reduce performance. Assuming that the publisher does not touch every piece, then $\delta<1$ for the following reason. Suppose that the attacker retrieves every piece of the content except one. If this piece was not touched by the publisher, the attacker can prove that the content was retrieved with $\delta<1$. Since we only account for the piece transfer costs, at least some of the time (when the attacker does not retrieve untouched pieces) $\delta<1$, which makes the expected value of all cases to be less than 1 . 


\subsection{Analysis of Puzzle Solving Strategies}

In what follows, we analyze the bandwidth cost of the malicious puzzle solving strategies described earlier, and show how to configure CAPnet's design parameters to achieve the desired $\delta$-bound. These parameters include the data piece size piece $_{\text {size }}$ and the number of puzzle rounds $R_{\text {puzzle }}$.

As mentioned previously, an attacker who wishes to solve the puzzle without retrieving all the data chunks will either exchange hashes or retrieve data pieces. By setting the piece size $\leq \frac{h_{\text {size }}}{m}$ one can ensure that the cost of transmitting a hash is no less than transmitting pieces. That is, even in the event when the piece provider has $m$ consecutive encrypted chunks, meaning that given one hash value the provider can process $m$ pieces in a puzzle round, transmitting a hash is more expensive than transmitting these $m$ pieces. In fact since the pieces may be used in multiple puzzle trials, it is better for the solver to retrieve them. For this reason, we focus on strategies that involve piece dissemination instead.

When retrieving data pieces to solve the challenge puzzle, we conjecture that the best strategy for the solver is to utilize its knowledge of the piece distribution across all trial puzzles. Initially, the solver must possess some piece of each encrypted data chunk to have a chance to solve the puzzle, since each round touches all encrypted data chunks. In order to get pieces from the honest caches, the client must download all double-layer encrypted chunks held by these caches. For malicious caches, the client can retrieve the individual pieces it desires. In selecting which pieces to retrieve, the best approach is to ask for the piece that gives the greatest chance of solving the puzzle. The solver can ask the piece provider to send the piece with the highest frequency among the remaining pieces, and then determine if it enables solving the challenge puzzle. This process continues until the solution is found. Assuming that retrieving the most popular missing piece is optimal, this is the optimal strategy for the malicious solver.

Recall that either the client or a cache $\left(\mathbf{C}_{m}\right)$ may play the role of the puzzle solver. If a cache is the puzzle solver, the client must still be the one to download the chunks from honest caches since the source IP of the request is checked. Hence, the client retrieval from honest caches is a fixed cost. Once this happens, it is more efficient for the party with the most content (either the client or $\mathbf{C}_{m}$ ) to act as the solver and get as few pieces as possible from the other party. This means that when $m<\frac{n}{2}$, the client will have a larger number of chunks than $\mathbf{C}_{m}$, thus, the client will be the puzzle solver. On the other hand, when $m>\frac{n}{2}$, $\mathbf{C}_{m}$ will be the puzzle solver asking the client to send pieces from the chunks it received from honest caches. When $m=\frac{n}{2}$, either party can be the puzzle solver.

Analyzing the bandwidth cost of the above strategy allows us to configure the number of puzzle rounds to obtain a specific $\delta$-bound. In order to do so, we compute the expected number of pieces $\mathbb{E}[Y]$ the colluding group will retrieve as a function of $R_{\text {puzzle }}$ and the number of malicious caches $m$. Then, we compute $\delta=\frac{\mathbb{E}[Y]}{n \cdot \text { pieces } \text { total }}$, after which we select $R_{\text {puzzle }}$ that satisfies the desired $\delta$-bound. To compute $\mathbb{E}[Y]$, we conduct simulations in which we mimic the above strategy and track the number of retrieved pieces. As an example, we consider the following setup, which we believe is similar to what is used in practical con- 
Table 2: The $\delta$-bound for various $m$ and $R_{\text {puzzle }}$ values, $n=6$ caches ( $R$ is $\left.R_{\text {puzzle }}\right)$. For $m<3$ the client is a more efficient puzzle solver, for $m>3 \mathbf{C}_{m}$ is a more efficient puzzle solver, $m=3$ is equivalent for each.

\begin{tabular}{|c|c|c|c|c|c|c|c|}
\hline & \multicolumn{3}{|c|}{ Client as solver } & \multirow{2}{*}{$\begin{array}{l}\text { Either } \\
\mathbf{3}\end{array}$} & \multicolumn{3}{|c|}{ Cache as solver } \\
\hline & 0 & 1 & 2 & & 4 & 5 & 6 \\
\hline 1 & 1 & $0.87 \pm 0.03$ & $0.78 \pm 0.06$ & $0.71 \pm 0.08$ & $0.45 \pm 0.06$ & $0.21 \pm 0.03$ & 0 \\
\hline 2 & 1 & $0.91 \pm 0.04$ & $0.86 \pm 0.06$ & $0.82 \pm 0.08$ & $0.52 \pm 0.06$ & $0.24 \pm 0.04$ & 0 \\
\hline 3 & 1 & $0.93 \pm 0.04$ & $0.9 \pm 0.05$ & $0.87 \pm 0.07$ & $0.57 \pm 0.05$ & $0.26 \pm 0.04$ & 0 \\
\hline 4 & 1 & $0.94 \pm 0.03$ & $0.92 \pm 0.05$ & $0.91 \pm 0.06$ & $0.59_{ \pm} 0.05$ & $0.28 \pm 0.03$ & 0 \\
\hline 5 & 1 & $0.95 \pm 0.03$ & $0.94 \pm 0.04$ & $0.93 \pm 0.04$ & $0.6 \pm 0.05$ & $0.29 \pm 0.03$ & 0 \\
\hline 6 & 1 & $0.96 \pm 0.03$ & $0.95 \pm 0.04$ & $0.94 \pm 0.04$ & $0.61 \pm 0.04$ & $0.29 \pm 0.03$ & 0 \\
\hline 7 & 1 & $0.96 \pm 0.02$ & $0.95 \pm 0.02$ & $0.95 \pm 0.04$ & $0.62 \pm 0.04$ & $0.3 \pm 0.03$ & 0 \\
\hline 8 & 1 & $0.97 \pm 0.02$ & $0.96 \pm 0.03$ & $0.95 \pm 0.03$ & $0.63 \pm 0.03$ & $0.3 \pm 0.02$ & 0 \\
\hline 9 & 1 & $0.97 \pm 0.02$ & $0.97 \pm 0.03$ & $0.96 \pm 0.03$ & $0.63 \pm 0.03$ & $0.3 \pm 0.02$ & 0 \\
\hline 10 & 1 & $0.97 \pm 0.02$ & $0.97 \pm 0.03$ & $0.97 \pm 0.03$ & $0.64 \pm 0.03$ & $0.31 \pm 0.02$ & 0 \\
\hline
\end{tabular}

tent distribution applications. We set chunk $_{\text {size }}=1 \mathrm{MB}$, and piece size $=16$ bytes, leading to pieces total $=2^{16}$ pieces. We have $R_{\text {puzzle }} \in\{1, \ldots, 10\}, n=6$, and $m \in\{1, \ldots, 6\}$. The simulations are repeated $10^{3}$ times, where $\mathbb{E}[Y]$, and consequently $\delta$, is computed as the average across all runs. We also report the standard deviation of our measurements. The computed $\delta$ values are found in Table 2.

As shown, as the number of rounds increases, $\delta$ increases. This is expected because a larger number of puzzle rounds means that the challenge puzzle requires a larger number of pieces to be solved. Consequently, the puzzle solver is expected to retrieve more content in order to find these pieces. On the other hand, $\delta$ decreases as the number of malicious caches increases for a fixed $R_{\text {puzzle }}$ value. Again, this is expected because more malicious caches makes the collusion more effective.

Note that scenarios where $\mathbf{C}_{m}$ is the solver have significantly lower $\delta$ values. This is because $\mathbf{C}_{m}$ already possesses the majority of the content that has been pooled at no cost (since we assume that metadata retrieval, such as keys, is free, pooling session keys costs no bandwidth). To solve the challenge puzzle, $\mathbf{C}_{m}$ only needs to retrieve the missing pieces from the client who has the data chunks from the honest caches.

Notice that as one would expect, once the piece provider and solver have their pieces it does not matter which party was a client or a cache originally. To see this, examine $\delta$ in Table 2. Notice that the points with $m=2$ and $m=4$ have a difference of 0.33 (within the margin of error). This is because the attacker either 
retrieves $\frac{2}{6}$ or $\frac{4}{6}$ of the content, a difference of 0.33 . However, in either case, a party with $\frac{4}{6}$ of the content acts as a solver while the party with $\frac{2}{6}$ provides pieces. Similar symmetries are found with $m=1$ and $m=5$ (a difference of 0.66 ) and $m=0$ and $m=6$ (a difference of 1). This illustrates that once a piece provider and solver have their content, the cost of solving the puzzle is uniform regardless of which party is the client and which is a cache.

\section{Evaluation}

In order to understand how CAPnet's security impacts efficiency, this section evaluates performance in the context of content distribution applications. Given that CAPnet imposes a minimal bandwidth cost to exchange a puzzle challenge and its solution, what is left to measure is its computational overhead. Towards this end, we conduct empirical experiments to answer the following specific questions:

- How fast can a publisher generate challenge puzzles?

- How quickly can a client solve these puzzles?

- How does the configuration of the design parameters affect these results?

- What do these numbers mean for a practical deployment?

The rest of this section describes our methodology and discusses the significance of the obtained results.

\subsection{Methodology}

To establish our benchmarks, we measured the rate, in puzzles per second, at which a publisher can generate challenge puzzles, and the rate at which a client can solve these puzzles. For the publisher, we considered the case of popular content that large numbers of clients routinely request in close time intervals. For the client, we computed the puzzle solving rate based on the average case, meaning that a client tries half the starting pieces in the first data chunk to find the solution.

Our experiments were conducted on a modest publisher server with an AMD Ryzen $32200 \mathrm{G}$ processor and $16 \mathrm{GiB}$ of memory, and a low-end client machine with an Intel Core i7-4600U processor and $8 \mathrm{GiB}$ of memory. Each puzzle generator and solver has been called at least 5,000,000 and 5,000 times, respectively. Unless otherwise mentioned, all graphs use $R_{\text {puzzle }}=5$, chunk $k_{\text {size }}=1 \mathrm{MB}$, $h_{\text {size }}=32$ bytes, and piece size $=16$ bytes. In addition, instead of reporting the puzzle rate for puzzle generator and solver, we compute the bitrate at which content can be requested using these puzzles. Despite both the client and publisher operations being embarrassingly parallelizable, we run each on a single core to show the per-core performance. 


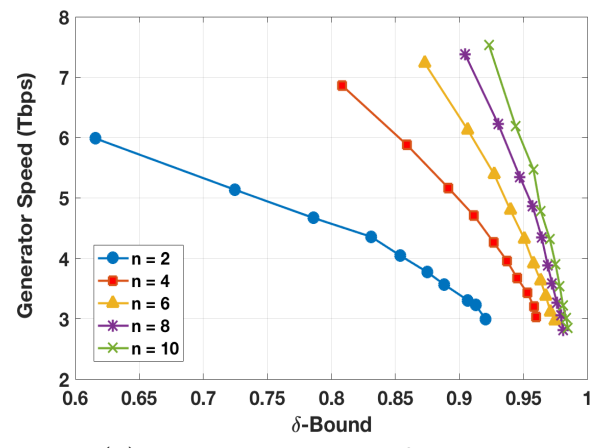

(a) Generator speed, $\delta$ effect.

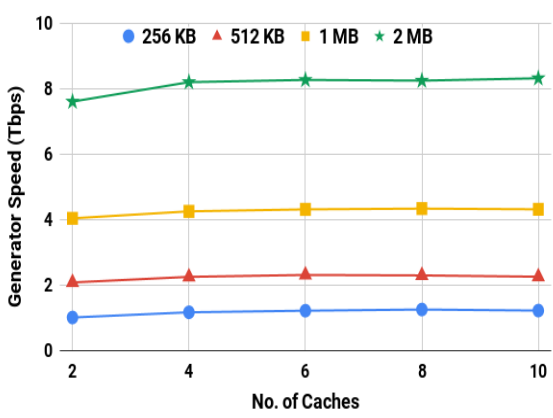

(b) Generator speed, chunk size $_{\text {effect }}$

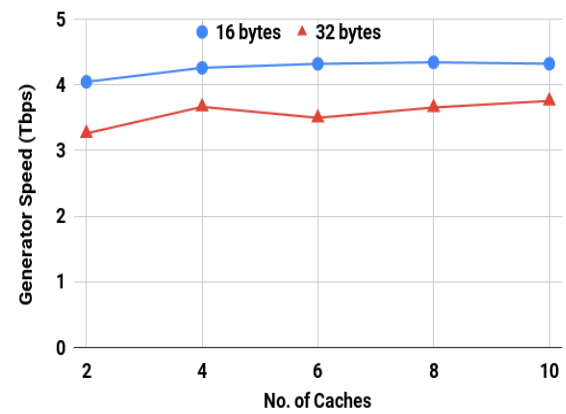

(c) Generator speed, piece $_{\text {size }}$ effect

Fig. 3: Generator speed for various configurations ( $n$ is the number of caches in a service session).

\section{$5.2 \quad$ Results}

Publisher's bitrate vs $\delta$. We begin by measuring the puzzle generation rate while varying the number of puzzle rounds $R_{\text {puzzle }}$ and number of caches $n$ with one malicious cache (Figure $3 \mathrm{a}$ ). As shown in the figure, we compute the $\delta$-bound value that corresponds to each $R_{\text {puzzle }}$ value. This produced a curve from 1 round (upper left point on each curve) to 10 rounds (lower right point). The bitrate decreases as $\delta$ increases because with larger $R_{\text {puzzle }}$ the publisher processes a larger number of pieces when preparing the challenge, which reduces the puzzle generation rate. On the other hand, an increased number of caches $n$ increases the throughput because more data is served per challenge puzzle. This factor also affects the $\delta$-bound of CAPnet. As shown in the figure, for larger $n$ the range $\delta$ gets larger for all $R_{\text {puzzle }}$ values. That is, the impact of having a malicious cache decreases when $n$ gets larger. This captures what happens in real life, where it will be harder for caches to collude effectively when there is a large number of caches per session. Based on the figure, setting $R_{\text {puzzle }} \geq 5$ in practice provides a reasonable $\delta$-bound for $n \geq 4(\delta \geq 0.93)$, with diminishing returns thereafter. 


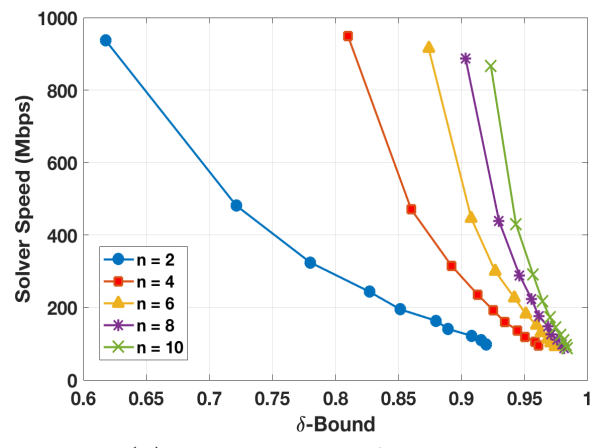

(a) Solver speed, $\delta$ effect.

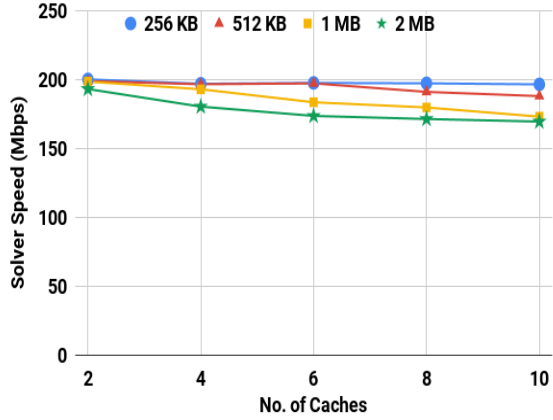

(b) Solver speed, chunk $_{\text {size }}$ effect

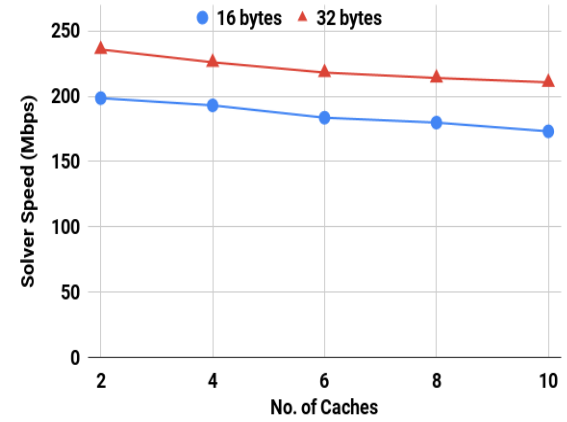

(c) Solver speed, piece $_{\text {size }}$ effect

Fig. 4: Solver speed for various configurations ( $n$ is the number of caches in a service session).

Client's bitrate vs $\delta$. Figure 4a shows the bitrate vs $\delta$-bound for the puzzle solver. As shown, for a fixed $R_{\text {puzzle }}$ value, the client's effective bandwidth is relatively uniform independent of the number of caches $n$. However, the $R_{p u z z l e}$ value, for a fixed $n$, has substantial impact on the effective bandwidth of a client. Given that the reported speed in the figure dwarfs the $5 \mathrm{Mbps}$ Netflix 1080p quality video rate [7], even using $R_{\text {puzzle }}=5$ (the $5^{\text {th }}$ point on each curve starting from the left), our modest client machine is able to watch dozens of $1080 \mathrm{p}$ videos concurrently. If higher performance is desired, then reducing $R_{\text {puzzle }}$, i.e., reducing $\delta$, provides drastically better performance, up to $900 \mathrm{Mbps}$, if needed.

How does chunk $_{\text {size }}$ impact client and publisher bitrates? We measured the puzzle generation and solving rates for various data chunk sizes and $n$ values (results are shown in Figures $3 \mathrm{~b}$ and $4 \mathrm{~b}$ ). The chunk $_{\text {size }}$ has a large effect on the performance of publishers, but minimal effect on client's performance. There are several reasons for this difference. The publisher has almost a fixed puzzle generation rate regardless of the chunk size, because it processes the same number of pieces for fixed $R_{\text {puzzle }}$, piece size $_{\text {, }}$ and $n$ values. Consequently, a larger chunk $_{\text {size }}$ makes the amount of content served per challenge puzzle 
larger. Alternatively, for a client the puzzle solving rate decreases with larger chunk $_{\text {size }}$ because the client has to try a larger number of trial puzzles. When computing the bitrate for some $n$ value, the low puzzle rates are multiplied by large chunk $_{\text {size }}$ and vice versa. For this reason the client bandwidth is somewhat similar for all chunk $k_{\text {size }}$ values.

How does piece $_{\text {size }}$ impact client and publisher bitrates? To understand how to set the piece size, we studied its effect on publisher (Figure 3c) and client (Figure 4c) performance. The publisher can generate enough puzzles to serve over 3 Tbps, regardless of the piece size. However, a piece size of 16 bytes is slightly more efficient because AES-CTR works on 16 byte blocks for encryption. In addition, a smaller piece size means that the publisher processes a smaller amount of content for a fixed number of pieces. The client, on the other hand, tends to benefit from larger piece sizes because they reduce the number of starting pieces, and hence, trial puzzles, a client has to compute. Given that a client with piece $_{\text {size }}=16$ byte already has a high throughput, and given that publishers are usually heavy-loaded entities, we recommend the use of piece size $=16$ bytes to boost publisher performance.

Contextualizing our results. To ground our results in real world numbers, we examined the customer demand from the popular content provider Netflix.com. Netflix serves 1080p video at a bitrate of approximately 5 Mbps [7]. As shown in Figure 3b, and taking $n=4$ caches, a publisher in our setup, using a single core machine, can generate puzzles for 136,000 requests per second, which translates to 544,000 data chunks per second. To understand this load, we look at a popular show, "House of Cards", where 5.4 million of its 83 million subscribers (as of 2015) watched at least one episode within a month of its release [5]. Since the report does not indicate how many of those views were concurrent, it is not possible to infer the exact peak load. However, our single core publisher supports concurrent viewing from 870,000 clients which is enough to support a simultaneous viewing peak from $1 / 6$ of the total views at any point during the first month.

As for the client, the previous results showed that for $R_{\text {puzzle }}=5$, on average our low-end client is able to solve enough puzzles to retrieve at least $170 \mathrm{Mbps}$ using a single core (Figure $4 \mathrm{~b}$ ). This is more than 34 times the rate required to retrieve the same popular 1080p video [7].

In summary, the previous results demonstrate that CAPnet is computationallylightweight. Its security in fighting cache accounting attacks is substantial $(\delta>$ 0.95 with generous attacker assumptions), even at bandwidth values that support a publisher serving thousands of clients or a client simultaneously watching dozens of 1080p videos. 


\section{Conclusions}

In this paper, we introduce CAPnet, a low-overhead solution to defend against cache accounting attacks in peer-assisted CDNs. CAPnet is the first system that forces malicious caches, even when colluding with clients, to expend substantial bandwidth to demonstrate that content was retrieved. This is done by introducing a cache accountability puzzle that provides strong protections even given unrealistically strong assumptions about the attacker's capabilities. For example, with a 5 round puzzle, if 3 malicious caches out of 6 total caches wish to perform a cache accounting attack, the colluding parties would retrieve on average more than 0.95 of the requested content $(\delta>0.95)$. We analyze the security of CAPnet, and show experimentally that it incurs a low computation cost, enabling a modest client to retrieve $170 \mathrm{Mbps}$ from a modest publisher serving several Tbps. This demonstrates the viability of employing our scheme in large scale content distribution applications.

\section{References}

1. Akamai. https://www.akamai.com/.

2. Akamai NetSession. https://www.akamai.com/us/en/products/media-delivery/ netsession-interface-overview.jsp.

3. Cisco Visual Networking Index: Forecast and Methodology, 2016-2021. https://www.cisco.com/c/en/us/solutions/collateral/service-provider/ visual-networking-index-vni/mobile-white-paper-c11-520862.html.

4. CloudFront. https://aws.amazon.com/cloudfront/.

5. House of Cards Viewers Stats. http://fortune.com/2016/03/05/house-ofcards-viewership/.

6. Netflix. https://www.netflix.com/.

7. Netflix Internet Connection Speed Recommendations. https://help.netflix.com/ en/node/306.

8. Swarmify. https://www.swarmify.com/.

9. Velocix. https://www.networks.nokia.com/products/velocix-media-deliveryplatform.

10. Youtube. https://www.youtube.com/.

11. Aditya, P., Zhao, M., Lin, Y., Haeberlen, A., Druschel, P., Maggs, B., And Wishon, B. Reliable client accounting for p2p-infrastructure hybrids. In NSDI'12 (2012).

12. Anjum, N., Karamshuk, D., Shikh-Bahaei, M., and Sastry, N. Survey on peer-assisted content delivery networks. Computer Networks 116 (2017).

13. Buyya, R., Pathan, M., And Vakali, A. Content delivery networks, vol. 9. Springer Science \& Business Media, 2008.

14. CASH, D., KüPÇÜ, A., AND WiChs, D. Dynamic proofs of retrievability via oblivious ram. Journal of Cryptology 30, 1 (2017).

15. Dziembowski, S., Faust, S., Kolmogorov, V., and Pietrzak, K. Proofs of space. In Crypto'15 (2015).

16. Erhay, C. C., KüPÇü, A., Papamanthou, C., And Tamassia, R. Dynamic provable data possession. ACM Transactions on Information and System Security 17, 4 (2015). 
17. Haeberlen, A., Kouznetsov, P., And Druschel, P. Peerreview: Practical accountability for distributed systems. ACM SIGOPS operating systems review 41, 6 (2007).

18. Jin, Y., Wen, Y., AND GUAN, K. Toward cost-efficient content placement in media cloud: modeling and analysis. IEEE Transactions on Multimedia 18, 5 (2016).

19. Lian, Q., Zhang, Z., Yang, M., Zhao, B. Y., Dai, Y., And Li, X. An empirical study of collusion behavior in the maze p2p file-sharing system. In ICDCS'0 7 (2007).

20. Michalakis, N., Soulé, R., And Grimm, R. Ensuring content integrity for untrusted peer-to-peer content distribution networks. In NSDI'07 (2007).

21. Nair, S. K., Zentveld, E., Crispo, B., and Tanenbaum, A. S. Floodgate: A micropayment incentivized $\mathrm{p} 2 \mathrm{p}$ content delivery network. In IEEE ICCCN'08 (2008).

22. Reiter, M. K., Sekar, V., Spensky, C., And Zhang, Z. Making peer-assisted content distribution robust to collusion using bandwidth puzzles. In ICISS'09 (2009).

23. Wang, C., Ren, K., Lou, W., And Li, J. Toward publicly auditable secure cloud data storage services. IEEE network 24, 4 (2010).

24. Zhao, M., Aditya, P., Chen, A., Lin, Y., Haeberlen, A., Druschel, P., Maggs, B., Wishon, B., And Ponec, M. Peer-assisted content distribution in akamai netsession. In $I M C^{\prime} 13$ (2013). 